\title{
COMPORTAMIENTO ASINTÓTICO DE ONDAS MAGNETO-ELÁSTICAS EN UN MEDIO CONDUCTIVO ACOTADO
}

\author{
Yolanda Silvia Santiago Ayala
}

Resumen.- Usando la Teoría de Semigrupo probamos la existencia de solución global de un modelo de evolución, la cual representa ondas magneto-elásticas en un medio conductivo acotado. También probamos que el sistema esta bien puesto y usando Sistemas Dinámicos y el Principio de Invariancia de La Salle mostramos que la energía asociada al sistema decae para cero cuando $t \rightarrow+\infty$.

Palabras claves.- Sistemas magneto-elásticos, Invariancia de La Salle, Decaimiento de la solución.

\section{ASYMPTHOTIC BEHAVIOR OF MAGNETO-ELASTIC WAVES IN A CONDUCTIVE BOUNDED ENVIRONMENT}

\begin{abstract}
Using semigroup theory we are going to proove the global existence solution of a evolution model, which represents the magneto-elastic waves in a boundary conductive media. Also, we are going to proove that the the sistem is well set and using Dinamic Systems and La Salle's Invariance Principle we are going to show that the energy asociated to the system decays to zero when $t \rightarrow+\infty$.
\end{abstract}

Key words.- Magneto-elastic Systems, La Salle's Invariance, Decayment of a solution.

\section{INTRODUCCION}

Considerando $\Omega \subset \mathbb{R}^{3}$, un dominio acotado de clase $C^{2}$.

$$
\begin{aligned}
& \rho u_{t t}-\mu \Delta u-(\lambda+\mu) \nabla \operatorname{divu}-\left(\frac{\mu_{o}}{4 \pi}\right) \operatorname{Roth} \times \tilde{H}=0 \\
& \beta h_{t}+\operatorname{Rot} \operatorname{Roth}-\beta \operatorname{Rot}\left[u_{t} \times \tilde{H}\right]=0 \\
& \operatorname{div} h=0
\end{aligned}
$$

\footnotetext{
${ }^{1}$ UNMSM, Facultad de Ciencias Matemáticas, e-mail: ysantiagoa@unmsm.edu.pe
} 
donde $u=\left(u_{1}, u_{2}, u_{3}\right) u_{j}=u_{j}(x, t)(x, t) \in \Omega \times \mathbb{R}^{+}$denota el desplazamiento, $h=\left(h_{1}, h_{2}, h_{3}\right), h_{j}=h_{j}(x, t)$ denota el campo magnético y $\tilde{H}=(0,0, c)$ fijo, con $c$ constante.

$\mu$ y $\lambda$ son las costantes de Lamé, con $\mu>0$ y $(\lambda+2 \mu)>0$. Las constantes $\rho, \mu_{o}$ y $\beta$ son positivas y son respectivamente, la densidad de masa, la permeabilidad magnética y el parámetro proporcional a la conductividad eléctrica.

Con condición de frontera para nuestro sistema

$$
\begin{aligned}
& u=0 \text { en } \partial \Omega \times \mathbb{R}^{+} \\
& h \cdot \eta=0 \text { en } \partial \Omega \times \mathbb{R}^{+} \\
& \operatorname{Rot} h \times \eta=0 \text { en } \partial \Omega \times \mathbb{R}^{+}
\end{aligned}
$$

donde $\eta=\eta(x)$ denota el vector normal unitario exterior en $x \in \partial \Omega$;

y condición Inicial

$$
u(x, 0)=u_{0}(x), \quad u_{t}(x, 0)=u_{1}(x), \quad h(x, 0)=h_{o}(x), x \in \Omega
$$

La energía asociada al sistema es

$$
E(t):=\frac{1}{2} \int_{\Omega}\left\{\rho\left|u_{t}\right|^{2}+\mu|\nabla u|^{2}+(\lambda+\mu)(\operatorname{div} u)^{2}+\frac{\mu_{o}}{4 \pi}|h|^{2}\right\} d x
$$

Nos planteamos la siguiente interrogante

- ¿Cual es el espacio natural $X$ donde se consigue existencia Global?

También, como para nuestro problema tenemos que $\frac{\partial E}{\partial t}=-\frac{\mu}{4 \pi \beta} \int_{\Omega}|R o t h|^{2} d x \leq 0$, entonces nos podemos preguntar

- ¿ $E(t) \longrightarrow 0$ cuando $t \rightarrow+\infty$ ?

La respuesta a estas interrogantes es positiva, determinamos el espacio $X$ donde existe la solución global y se consigue probar la convergencia a cero de la energía, cuando $t$ va para infinito.

Abordamos la existencia de solución usando la Teoría de Semigrupos y ana-lizamos el comportamiento asintótico haciendo uso del Principio de Invariancia de La Salle. 
Es importante destacar que hace 39 años Dafermos [4] estudió el siguiente problema

$$
\begin{aligned}
\rho u_{t t}-\mu \Delta u-(\lambda+\mu) \nabla \operatorname{divu}+\nabla \theta & =0 \\
\beta \theta_{t}-\Delta \theta-\beta \operatorname{div} u_{t} & =0
\end{aligned}
$$

donde $u=\left(u_{1}, u_{2}, u_{3}\right), \theta=\theta(x, t) \in \mathbb{R}$. La energía $\tilde{E}$ asociada a este sistema, satisface $\frac{\partial \tilde{E}}{\partial t}=-\int_{\Omega}|\nabla \theta|^{2} d x \leq 0$. Entonces surgen dos problemas:

1. $\tilde{E}(t) \rightarrow 0$ cuando $t \rightarrow+\infty$.

2. $\tilde{E}(t)$ decae con tasa uniforme (exponencial ó polinomialmente).

Dafermos probó que existe $\Omega$ tal que $\tilde{E}(t)$ no converge a cero. En verdad depende de la geometría de $\Omega$. Por ejemplo si $\Omega$ es una bola, $\tilde{E}(t)$ puede no converger a cero.

Además, Dafermos afirmó que 1. vale en ciertas regiones debido al Principio de Invariancia de La Salle [15].

Por otro lado, Koch [7] afirmó que se puede tener $\Omega$ satisfaciendo 1. y no 2 .

De ahí que esto motiva usar en este trabajo el Principio de Invariancia de La Salle.

Debemos citar los trabajos de Perla Menzala and Zuazua [17], Andreou and Dassios [1], Avalos and Lasiecka [2], Y. Santiago and J. Rivera [14], e Y. Santiago [15], que ilustran métodos de como abordar el estudio del comportamiento asintótico de un problema de evolución.

\section{PRINCIPALES RESULTADOS}

Enunciamos nuestro resultado de existencia de solución global.

Teorema 2.1 Considerando el sistema (1.1), (1.2), (1.3), con la constante magnética $\tilde{H}$ y los parámetros $\lambda, \mu, \mu_{0}$ y $\beta$ definidos inicialmente.

Sea $\left(u_{0}, u_{1}, h_{0}\right) \in X:=\left[H_{0}^{1}(\Omega)\right]^{3} \times\left[L^{2}(\Omega)\right]^{3} \times Y$,

donde $Y:=\left\{f \in\left[L^{2}(\Omega)\right]^{3}\right.$, div $f=0$ en $\Omega, f \cdot \eta=0$ en $\left.\partial \Omega\right\}$.

Entonces el problema (1.1)-(1.3) es GLOBALMENTE BIEN PUESTO y la solución débil $\left(u, u_{t}, h\right) \in C([0, \infty), X)$.

Además, si $\left(u_{o}, u_{1}, h_{0}\right) \in D(A)$, la solución $\left(u, u_{t}, h\right) \in C([0, \infty), D(A)) \cap C^{1}([0, \infty), X)$ 
verifica (1.1)-(1.3) y satisface:

$$
\forall t \geq 0, \quad\left\|\left(u(t), u_{t}(t), h(t) / \alpha\right)\right\|_{D(A)} \leq\left\|\left(u_{0}, u_{1}, h_{0} / \alpha\right)\right\|_{D(A)}
$$

i.e.

$$
E^{\prime}(t)=-\frac{\mu_{0}}{4 \pi \beta} \int_{\Omega}|\operatorname{Rot} h|^{2} d x, t \geq 0
$$

En el estudio del comportamiento asintótico de la solución se logra mostrar el siguiente resultado.

Teorema 2.2 (Resultado Principal) Sea $\Omega$ un dominio suave, simplemente conexo, acotado de $\mathbb{R}^{3}$. Entonces, la solución de energía finita del sistema (1.1)-(1.3) es tal que $E(t) \rightarrow 0$ cuando $t \rightarrow+\infty$.

\section{EXISTENCIA DE SOLUCIÓN GLOBAL}

El sistema (1.1)-(1.3) es equivalente al siguiente Problema de Cauchy Abstracto,

$$
\mid \begin{aligned}
U_{t} & =A U, \quad t>0 \\
U(0) & =U_{o}=\left(u_{0}, u_{1}, \frac{h_{o}}{\alpha}\right) \in X
\end{aligned}
$$

donde

$$
U(t)=\left(u, u_{t}, h / \alpha\right), \alpha:=\sqrt{\frac{4 \pi \rho}{\mu_{o}}} .
$$

y

$$
X:=\left[H_{0}^{1}(\Omega)\right]^{3} \times\left[L^{2}(\Omega)\right]^{3} \times Y
$$

donde el espacio $Y$ es

$$
Y:=\left\{f \in\left[L^{2}(\Omega)\right]^{3}, \operatorname{div} f=0 \text { en } \Omega, f \cdot \eta=0 \text { en } \partial \Omega\right\} \text {. }
$$

Las normas consideradas son respectivamente:

$$
\begin{gathered}
\|g\|_{Y}:=\left(\int_{\Omega}|g|^{2} d x\right)^{\frac{1}{2}}=\left(\sum_{i=1}^{3} \int_{\Omega}\left|g_{i}\right|^{2} d x\right)^{\frac{1}{2}}, \\
\|f\|_{\left[H_{0}^{1}(\Omega)\right]^{3}}:=\left[\int_{\Omega} \mu|\nabla f|^{2}+(\lambda+\mu)|\operatorname{div} f|^{2} d x\right]^{\frac{1}{2}} .
\end{gathered}
$$


Se observa que $\|\cdot\|_{\left[H_{0}^{1}(\Omega)\right]^{3}}$ es equivalente a $\|\cdot\|_{\left[H^{1}(\Omega)\right]^{3}}$ (debido a la desigualdad de Korn, ver [9] ).

El Operador $A$ esta definido por

$$
A:=\left[\begin{array}{ccc}
0 & I & 0 \\
-A_{1} & 0 & B \\
0 & C & -A_{2}
\end{array}\right]
$$

donde

$$
A_{1} u:=-\frac{\mu}{\rho} \Delta u-\frac{(\lambda+\mu)}{r h o} \nabla \operatorname{div} u, \quad \text { para } u \in D\left(A_{1}\right)
$$

y $A_{1}: D\left(A_{1}\right) \rightarrow\left[L^{2}(\Omega)\right]^{3}$ con $D\left(A_{1}\right):=\left[H^{2}(\Omega) \cap H_{0}^{1}(\Omega)\right]^{3} \subset\left[L^{2}(\Omega)\right]^{3}$. Así, $A_{1}$ es un operador no acotado y es un isomorfismo de $\left[H_{0}^{1}(\Omega)\right]^{3}$ en su dual $\left[H^{-1}(\Omega)\right]^{3}$.

$$
B h:=\frac{\mu_{0} \alpha}{4 \pi \rho} \operatorname{Rot} h \times \tilde{H} \quad \text { para } h \in D(B)
$$

y $B: D(B) \rightarrow\left[L^{2}(\Omega)\right]^{3}$ con $D(B):=\left\{h \in Y,(R o t) \times \tilde{H} \in\left[L^{2}(\Omega)\right]^{3}\right\} \subset Y$. Así, $B$ es un operador no acotado.

$$
A_{2} h:=\frac{1}{\beta} \operatorname{Rot} \operatorname{Rot} h \quad \text { para } h \in D\left(A_{2}\right)
$$

y $A_{2}: D\left(A_{2}\right) \rightarrow Y$ con $D\left(A_{2}\right):=\left\{g \in\left[H^{2}(\Omega)\right]^{3} \cap Y, \operatorname{Rot} g \times \eta=0\right.$ en $\left.\partial \Omega\right\} \subset Y$. Así, $A_{2}$ es un operador no acotado.

$$
C v:=\frac{1}{\alpha} \operatorname{Rot}(v \times \tilde{H}) \text { para } v \in D(C)
$$

y $C: D(C) \rightarrow Y$ con $D(C):=\left\{v \in\left[L^{2}(\Omega)\right]^{3}, \operatorname{Rot}(v \times \tilde{H}) \in Y\right\} \subset\left[L^{2}(\Omega)\right]^{3}$. Así, $C$ es un operador no acotado.

El dominio del operador $A$ es

$$
D(A):=\left[H^{2}(\Omega) \cap H_{0}^{1}(\Omega)\right]^{3} \times\left[H_{0}^{1}(\Omega)\right]^{3} \times D\left(A_{2}\right) \subset X .
$$


verifica (1.1)-(1.3) y satisface:

$$
\forall t \geq 0, \quad\left\|\left(u(t), u_{t}(t), h(t) / \alpha\right)\right\|_{D(A)} \leq\left\|\left(u_{0}, u_{1}, h_{0} / \alpha\right)\right\|_{D(A)}
$$

i.e.

$$
E^{\prime}(t)=-\frac{\mu_{0}}{4 \pi \beta} \int_{\Omega}|\operatorname{Rot} h|^{2} d x, t \geq 0
$$

En el estudio del comportamiento asintótico de la solución se logra mostrar el siguiente resultado.

Teorema 2.2 (Resultado Principal) Sea $\Omega$ un dominio suave, simplemente conexo, acotado de $\mathbb{R}^{3}$. Entonces, la solución de energía finita del sistema (1.1)-(1.3) es tal que $E(t) \rightarrow 0$ cuando $t \rightarrow+\infty$.

\section{EXISTENCIA DE SOLUCIÓN GLOBAL}

El sistema (1.1)-(1.3) es equivalente al siguiente Problema de Cauchy Abstracto,

$$
\mid \begin{aligned}
U_{t} & =A U, \quad t>0 \\
U(0) & =U_{o}=\left(u_{0}, u_{1}, \frac{h_{o}}{\alpha}\right) \in X
\end{aligned}
$$

donde

$$
U(t)=\left(u, u_{t}, h / \alpha\right), \alpha:=\sqrt{\frac{4 \pi \rho}{\mu_{o}}}
$$

y

$$
X:=\left[H_{0}^{1}(\Omega)\right]^{3} \times\left[L^{2}(\Omega)\right]^{3} \times Y
$$

donde el espacio $Y$ es

$$
Y:=\left\{f \in\left[L^{2}(\Omega)\right]^{3}, \operatorname{div} f=0 \text { en } \Omega, f \cdot \eta=0 \text { en } \partial \Omega\right\}
$$

Las normas consideradas son respectivamente:

$$
\begin{gathered}
\|g\|_{Y}:=\left(\int_{\Omega}|g|^{2} d x\right)^{\frac{1}{2}}=\left(\sum_{i=1}^{3} \int_{\Omega}\left|g_{i}\right|^{2} d x\right)^{\frac{1}{2}}, \\
\|f\|_{\left[H_{0}^{1}(\Omega)\right]^{3}}:=\left[\int_{\Omega} \mu|\nabla f|^{2}+(\lambda+\mu)|\operatorname{div} f|^{2} d x\right]^{\frac{1}{2}} .
\end{gathered}
$$




$$
\begin{aligned}
& \text { Prueba.- Sea } U=\left(\begin{array}{c}
u \\
v \\
w
\end{array}\right) \in D(A) \text { entonces tenemos } \\
&<A U, U>_{X}=<v, u>_{\left(H_{0}^{1}\right)^{3}}+<-A_{1} u+B w, v>_{\left(L^{2}\right)^{3}}+<C v-A_{2} w, w>_{Y} \\
&=\left.<v, u>_{\left(H_{0}^{1}\right)^{3}}-<A_{1} u, v>_{\left(L^{2}\right)^{3}}+<B w, v\right\rangle_{\left(L^{2}\right)^{3}}+ \\
&<C v, w>_{Y}-<A_{2} w, w>_{Y} \\
&=-\frac{1}{\beta} \int_{\Omega}|\operatorname{Rot} w|^{2} d x \leq 0 .
\end{aligned}
$$

Aquí hemos usado respectivamente las siguientes identidades:

- $<B w, v>_{\left(L^{2}\right)^{3}}=-<C v, w>_{Y}$, cuando $(u, v, w) \in D(A)$

- $<A_{2} w, w>_{Y}=\frac{1}{\beta} \int_{\Omega}|\operatorname{Rot} w|^{2} d x$.

- $<v, u>_{\left(H_{0}^{1}\right)^{3}}=<A_{1} u, v>_{\left(L^{2}\right)^{3}}$

Desde que $A$ es maximal si y solamente si $\operatorname{Im}(I-A)=X$, procedemos a probar que $\operatorname{Im}(I-A)=X$.

$$
\begin{gathered}
\text { Sea } f=\left(\begin{array}{c}
f_{1} \\
f_{2} \\
f_{3}
\end{array}\right) \in X \text {, probaremos que existe }\left(\begin{array}{c}
u \\
v \\
w
\end{array}\right) \in D(A) \text { tal que } \\
(I-A)\left(\begin{array}{c}
u \\
v \\
w
\end{array}\right)=\left(\begin{array}{c}
f_{1} \\
f_{2} \\
f_{3}
\end{array}\right)
\end{gathered}
$$

(1) es equivalente al sistema

$$
\begin{aligned}
u-v & =f_{1} \\
v+A_{1} u-B w & =f_{2} \\
w-C v+A_{2} w & =f_{3}
\end{aligned}
$$

(3.2) implica

$$
v=u-f_{1}
$$


Substituyendo (3.5) en (3.3) tenemos

$$
\begin{aligned}
& u-f_{1}+A_{1} u-B w=f_{2} \\
& \text { i.e. } u+A_{1} u-B w=f_{2}+f_{1}:=g_{1} \in\left[L^{2}(\Omega)\right]^{3}
\end{aligned}
$$

Substituyendo (3.5) en (3.4) tenemos

$$
\begin{aligned}
w-C\left\{u-f_{1}\right\}+A_{2} w & =f_{3} \\
w-C u+C f_{1}+A_{2} w & =f_{3} \\
-C u+w+A_{2} w & =f_{3}-C f_{1}:=g_{2}
\end{aligned}
$$

Así, basta resolver (3.6) y (3.7), es decir:

$$
(*) \mid \begin{aligned}
u+A_{1} u-B w & =g_{1} \in\left[L^{2}(\Omega)\right]^{3} \\
-C u+w+A_{2} w & =g_{2} \in Y
\end{aligned}
$$

Observación 3.1 Si $F=\left(f_{1}, f_{2}, f_{3}\right) \in C^{2}$ entonces div Rot $F=0$.

Observación 3.2 Si $F \in\left[L^{2}(\Omega)\right]^{3}$ entonces div $\operatorname{Rot} F=0$.

Lema $3.2\left[H_{0}^{1}(\Omega)\right]^{3}$ está inmerso continuamente en

$$
D(C):=\left\{v \in\left[L^{2}(\Omega)\right]^{3}, \quad \operatorname{Rot}(V \times \tilde{H}) \in Y\right\}
$$

Introducimos la siguiente forma bilineal en $L:=\left[H_{0}^{1}(\Omega)\right]^{3} \times V$, donde

$$
\begin{gathered}
V:=\left\{w \in Y, \operatorname{Rot} w \in\left[L^{2}(\Omega)\right]^{3}\right\} \\
a(., .): L \times L \longrightarrow \mathbb{R} \\
a((u, w),(\tilde{u}, \tilde{w})):=\int_{\Omega}\left\{u \tilde{u}+\mu \nabla u \cdot \nabla \tilde{u}+(\lambda+\mu) \operatorname{div} u \operatorname{div} \tilde{u}+w \tilde{w}+\frac{1}{\beta} \operatorname{Rot} w \cdot \operatorname{Rot} \tilde{w}\right\} d x \\
-<B w, \tilde{u}>_{\left(L^{2}\right)^{3}}-<C u, \tilde{w}>_{Y}
\end{gathered}
$$

Esta forma bilineal es continua y coerciva en $L$, entonces usando el Teorema de 
Lax Milgram tenemos que $\exists !(u, w) \in L$ tal que $a((u, w),(\tilde{u}, \tilde{w}))=<\left(g_{1}, g_{2}\right),(\tilde{u}, \tilde{w})>_{L}$, $\forall(\tilde{u}, \tilde{w}) \in L$

Así, $(u, w)$ es solución débil de $(*)$.

Observación 3.3 Si $w \in V$ entonces Bw $=\operatorname{Rot} w \times \tilde{H} \in\left[L^{2}(\Omega)\right]^{3}$

Por Regularidad elíptica deducimos que $u \in D\left(A_{1}\right)=\left(H^{2} \cap H_{0}^{1}\right)^{3}$.

Análogamente probamos que $w \in D\left(A_{2}\right)$.

Siendo $A$ disipativo y maximal, usamos el Teorema de Lumer Phillips para concluir que $A$ es el generador infinitesimal de un semigrupo de contracciones en $X$. Así podemos enunciar nuestro resultado principal de existencia de solución global.

Teorema 3.1 Considerando el sistema (1.1), (1.2), (1.3) donde al inicio indicamos como son la constante magnética $\tilde{H}$ y los parámetros $\lambda, \mu, \mu_{0}$ y $\beta$.

Sea $\left(u_{0}, u_{1}, h_{0}\right) \in X:=\left[H_{0}^{1}\right]^{3} \times\left[L^{2}\right]^{3} \times Y$,

donde $Y:=\left\{f \in\left[L^{2}\right]^{3}\right.$, div $f=0$ en $\Omega, f \cdot \eta=0$ en $\left.\partial \Omega\right\}$.

Entonces el problema (1.1)-(1.3) es GLOBALMENTE BIEN PUESTO y la solución débil $\left(u, u_{t}, h\right) \in C([0, \infty), X)$.

Además, si $\left(u_{o}, u_{1}, h_{0}\right) \in D(A)$, la solución $\left(u, u_{t}, h\right) \in C([0, \infty), D(A)) \cap C^{1}([0, \infty), X)$ verifica (1.1)-(1.3) y satisface:

$$
\forall t \geq 0, \quad\left\|\left(u(t), u_{t}(t), h(t) / \alpha\right)\right\|_{D(A)} \leq\left\|\left(u_{0}, u_{1}, h_{0} / \alpha\right)\right\|_{D(A)}
$$

i.e. $E^{\prime}(t)=-\frac{\mu_{0}}{4 \pi \beta} \int_{\Omega}|\operatorname{Rot} h|^{2} d x, t \geq 0$.

En el cálculo de $E^{\prime}(t)$ hemos usado la Identidad de Green, que el Rotacional es Autoadjunto en $Y, \mathrm{y}$

$$
\begin{aligned}
A \cdot(B \times C) & =B \cdot(C \times A) \\
& =-B \cdot(A \times C) .
\end{aligned}
$$

Para abordar el estudio del comportamiento asintótico, precisamos del Principio de Invariancia de La Salle, ver [5], [18]. Para fijar ideas, por ese motivo introducimos las dos siguientes secciones. 


\section{SISTEMAS DINÁMICOS ABSTRACTOS}

Sea $(X, d)$ un espacio métrico completo,

Definición 4.1 Sea $S_{t}: X \longrightarrow X . t \geq 0$, diremos que $\left\{S_{t}\right\}_{t \geq 0}$, es un sistema dinámico en $X$ si satisface:

a) $S_{t} \in C(X, X), \forall t \geq 0$.

b) $S_{0}=I_{d}$.

c) $S_{t+s}=S_{t} S_{s}, \forall s, t \geq 0$.

d) $\forall x \in X$, la aplicación $: t \longrightarrow S_{t} x$ es continua de $[0, \infty) \longrightarrow X$.

Observación 4.1 Sea $F \subset X$ un conjunto cerrado (i.e. $(F, d)$ es completo) tal que $S_{t} F \subset F$, entonces $\left\{\left.S_{t}\right|_{F}\right\}$ es un sistema dinámico en $(F, d)$.

Definición 4.2 Sea $x \in X, \bigcup_{t \geq 0}\left\{S_{t} x\right\}=\gamma(x)=$ "ORBITA"

Observación 4.2 Si $X$ es un espacio vectorial con $S_{t}$ lineal, $S_{t}$ es un Semigrupo lineal.

Tenemos la siguiente afirmación

\section{PROBLEMA}

Dado $x \in X$ existe $\Theta(x) \subset X$ tal que $d\left(S_{t} x, \Theta(x)\right) \longrightarrow 0$ cuando $t \rightarrow+\infty$. Por eso introducimos la siguiente definición

Definición 4.3 Sea $x \in X$, definamos el conjunto Omega-límite como

$$
\omega(x):=\left\{y \in X, \exists t_{n} \rightarrow+\infty, S_{t_{n}} x \longrightarrow y \text { cuando } t_{n} \rightarrow+\infty\right\}
$$

Observación 4.3 Podemos fácilmente analizar $\omega(x)$ de sistemas bidimensionales simples. 
Proposición 4.1 Sea $x \in X$, entonces $\omega(x)=\cap_{s \geq 0} \overline{\cup_{t \geq s}\left\{S_{t} x\right\}}$

i.e. $\omega(x)$ es cerrado.

Observe a su vez que $\omega(x) \subset \overline{U_{t \geq s}\left\{S_{t} x\right\}}=\overline{\gamma(x)}$.

Proposición 4.2 Sea $x \in X$, entonces $\forall t \geq 0$ se satisfacen los siguientes enunciados:

a) $\omega\left(S_{t} x\right)=\omega(x)$.

b) $S_{t}(\omega(x)) \subset w(x)$.

c) Si $\gamma(x)$ es relativamente compacto en $X$ entonces $\emptyset \neq \omega(x)=S_{t}(\omega(x))$ y $\omega(x)$ es compacto.

Proposición 4.3 Sea $x \in X$ y $\gamma(x)$ relativamente compacto en $X$ entonces

a) $\omega(x)$ es compacto $y$ conexo en $X$.

b) $d\left(S_{t} x, \omega(x)\right) \longrightarrow 0$ cuando $t \rightarrow+\infty$.

5. FUNCIONES DE LYAPUNOV Y EL PRINCIPIO DE INVARIANCIA DE LA SALLE

Definición 5.1 Sea $\mathcal{L} \in C(X, \mathbb{R}),(X, d)$ un espacio métrico completo. Diremos que $\mathcal{L}$ es una función de Lyapunov para $\left\{S_{t}\right\}_{t \geq 0}$ si $\mathcal{L}\left(S_{t} x\right) \leq \mathcal{L}(x), \forall x \in X, \forall t \geq 0$.

Observación $5.1: t \longrightarrow \mathcal{L}\left(S_{t} x\right)$ es decreciente.

Teorema 5.1 (PRINCIPIO DE INVARIANCIA DE LA SALLE) Sea $\mathcal{L}$ una función de Lyapunov para $\left\{S_{t}\right\}_{t \geq 0}$. Sea $x \in X$ tal que $\gamma(x)$ es relativamente compacto en $X$, entonces:

a) Existe $\lim _{t \rightarrow+\infty} \mathcal{L}\left(S_{t} x\right)=L$

b) $L=\mathcal{L}(y), \forall y \in \omega(x)$.

Definición 5.2 Sea $x \in X,\left\{S_{t}\right\}_{t \geq 0}$ un sistema dinámico en $X$, diremos que $x_{e} \in X$ es un punto de equilibrio si $S_{t} x_{e}=x_{e}, \forall t \geq 0$.

Observación $5.2 \omega\left(x_{e}\right)=\left\{x_{e}\right\}$. 
Definición 5.3 Sea $F \subset X, F$ es invariante por la familia $\left\{S_{t}\right\}_{t \geq 0}$ si existe una aplicación $f: \mathbb{R}^{+} \times F \longrightarrow F$ tal que

$f(0, x)=x, \forall x \in F$.

$f(t+s, x)=S_{t} f(s, x), \forall x \in F, \forall s, t \in \mathbb{R}^{+}$

Observación 5.3 Si $x:=x_{e}$ es punto de equilibrio, entonces $\gamma\left(x_{e}\right)=\left\{x_{e}\right\}$ es invariante.

Observación $5.4 \mathrm{Si}\left\{S_{t} x_{o}\right\}_{t \geq 0}$ es periódico en $t$, con periódo $T$, entonces $\gamma\left(x_{o}\right)$ es invariante.

Definición $5.4 \mathcal{L}$ es función de Lyapunov estricta si $\mathcal{L}\left(S_{t} x\right)=\mathcal{L}(x), \forall t \geq 0$ implica que $x$ sea punto de equilibrio.

Teorema 5.2 Sea $\mathcal{L}$ función de Lyapunov estricta para $\left\{S_{t}\right\}_{t \geq 0}$. Sea $x \in X$ tal que $\overline{\gamma(x)}$ es compacto, defina $\mathcal{E}$ como el conjunto de puntos de equilibrio en $X$. Entonces:

1) $\mathcal{E}$ es cerrado y $\mathcal{E} \neq \emptyset$.

2) $d\left(S_{t} x, \mathcal{E}\right) \longrightarrow 0$ cuando $t \rightarrow+\infty$. Esto quiere decir que "E atrae a todas las orbitas".

Corolario 5.1 Sea $\mathcal{L}$ función de Lyapunov estricta para $\left\{S_{t}\right\}_{t \geq 0}$. Sea $x \in X$ tal que $\overline{\gamma(x)}$ es compacto.

Sea $L=\lim _{t \rightarrow+\infty} \mathcal{L}\left(S_{t} x\right)$ y $\mathcal{E}_{L}:=\{x \in \mathcal{E}$ tal que $\mathcal{L}(x)=L\}$. Entonces:

a) $\mathcal{E}_{L}$ es cerrado y $\mathcal{E}_{L} \neq \emptyset$.

b) $d\left(S_{t} x, \mathcal{E}_{L}\right) \longrightarrow 0$ cuando $t \rightarrow+\infty$.

c) Si $\mathcal{E}_{L}$ es discreto, entonces existe $y \in \mathcal{E}_{L}$ tal que $S_{t} x \longrightarrow y$ cuando $t \rightarrow+\infty$.

\section{COMPORTAMIENTO ASINTÓTICO DE LA SOLUCIÓN}

Si $\left(u_{0}, u_{1}, h_{0}\right) \in D(A)$ entonces $\left(u, u_{t}, h\right)$ está acotada en $D(A)$. Usando Inmersiones de Sobolev, se deduce que la trayectoria $\left(u, u_{t}, h\right)$ es relativamente compacto en $X$. Así,

$$
\emptyset \neq \underbrace{w\left(\left(u_{o}, u_{1}, h_{o}\right)\right)}_{\text {conjunto } w \text {-límite }}:=\begin{aligned}
& \left\{P_{0}:=\left(\tilde{u}_{0}, \tilde{u}_{1}, \tilde{h}_{o}\right) \in X \text { tal que } \exists\left(t_{n}\right), t_{n} \rightarrow+\infty,\right. \\
& \left.\left(u\left(t_{n}\right), u_{t}\left(t_{n}\right), h\left(t_{n}\right)\right) \rightarrow P_{0}, \text { cuando } n \rightarrow+\infty\right\}
\end{aligned}
$$


Considerando a $E(t)$ como el Funcional de Lyapunov, que decrece a lo largo de trayectorias con disipación $-\frac{\mu_{0}}{4 \pi \rho} \int_{\Omega}|\operatorname{Rot} h|^{2} d x$, y usando el Principio de Invariancia de La Salle, tenemos que

$w\left(\left(u_{o}, u_{1}, h_{o}\right)\right) \subset F:=\left\{\left(\tilde{u}_{0}, \tilde{u}_{1}, \tilde{h}_{o}\right) \in X\right.$ tal que la solución de (1.1)-(1.3) implica

$$
\left.\operatorname{Rot} h=0 \text { en } \Omega \times \mathbb{R}^{+}\right\}
$$

Para poder concluir que las soluciones de (1.1)-(1.3) convergen a cero en $X$, cuando $t \rightarrow+\infty$, basta analizar si $F$ es o no $\{(0,0,0)\}$.

Vamos ha suponer que $\Omega$ es simplemente conexo.

Analizaremos la estructura de $F$.

Rot $h=0$ en $\Omega \times \mathbb{R}^{+}, \Omega$ es simplemente conexo., entonces $h=\nabla P$, para algún $P$.

$\operatorname{div} h=0$ en $\Omega \times \mathbb{R}^{+}$.

Por lo tanto, $\operatorname{div} h=\operatorname{div} \nabla P=\Delta P=0$ en $\Omega \times \mathbb{R}^{+}$.

Que $h \in Y$ implica $h \cdot \eta=0$ en $\partial \Omega \times \mathbb{R}^{+}$.

Por lo tanto, $h \cdot \eta=\nabla P \cdot \eta=\frac{\partial P}{\partial \eta}=0$ en $\partial \Omega \times \mathbb{R}^{+}$.

Así, $P$ es constante, i.e. $h=\nabla P=0$ en $\Omega \times \mathbb{R}^{+}$.

(1.1) queda:

$$
\rho u_{t t}-\mu \Delta u-(\lambda+\mu) \nabla \operatorname{div} u=0
$$

(1.2) queda:

$$
\operatorname{Rot}\left[u_{t} \times \tilde{H}\right]=0 \quad \text { en } \Omega \times \mathbb{R}^{+}
$$

Por lo tanto, $F$ puede ser caracterizado como el subespacio de dato inicial $\left(u_{0}, u_{1}, h_{0}\right)$ de energia finita que satisface (a), (b) y (c).

(a) $h=0$ en $\Omega \times \mathbb{R}^{+}$.

(b) $u$ es solución del Sistema de Lamé:

$$
\mid \begin{aligned}
& \rho u_{t t}-\mu \Delta u-(\lambda+\mu) \nabla \operatorname{div} u=0 \text { en } \Omega \times \mathbb{R}^{+} \\
& u=0 \text { en } \partial \Omega \times \mathbb{R}^{+} \\
& u(x, 0)=u_{0}(x), \quad u_{t}(x, 0)=u_{1}(x) \text { en } \Omega
\end{aligned}
$$

(c) u satisface:

$$
\operatorname{Rot}\left[u_{t} \times \tilde{H}\right]=0 \quad \text { en } \Omega \times \mathbb{R}^{+}
$$

Finalmente, todo se reduce a probar que $u \equiv 0$. 
Usamos argumentos de C. Dafermos (1968) "On the existence and asymptotic stability of solutions to the equations of linear thermoelasticity", ver [4].

Expansión en series de Fourier de la Solución de (b) en la base de autofunciones del Operador Elíptico de Lamé $\tilde{L}$ y las propiedades de la exponencial compleja:

$$
\begin{aligned}
& \lim _{T \rightarrow+\infty} \frac{1}{T} \int_{0}^{T} e^{i \alpha t} e^{i \beta t} d t=\delta_{\alpha, \beta} \quad, \alpha, \beta \in \mathbb{R} \\
& \tilde{L}=\mu \Delta u+(\lambda+\mu) \nabla \operatorname{div} u \\
& \mid \begin{array}{l}
-\tilde{L} w_{j}=\lambda_{j}^{2} w_{j}, \quad x \in \Omega \\
w_{j}=0 \text { en } \partial \Omega \\
w_{j} \in H_{0}^{1}(\Omega)
\end{array}
\end{aligned}
$$

Por otro lado,

(b) y (c) tienen una solución no trivial

Existe una autofunción no-trivial $w=w(x) \in\left(H_{0}^{1}(\Omega)\right)^{3}$ del sistema de Lamé:

$$
(I) \mid \begin{aligned}
& -\mu \Delta w-(\lambda+\mu) \nabla \operatorname{div} w=\rho \gamma^{2} w \text { en } \Omega \\
& w=0 \text { en } \partial \Omega
\end{aligned}
$$

tal que

$$
\begin{gathered}
(I I) \mid \operatorname{Rot}[w \times \tilde{H}]=0 \text { en } \Omega \\
\Downarrow \\
F \supset \text { elementos no triviales. }
\end{gathered}
$$

Teorema 6.1 (Resultado Principal) Son equivalentes los siguientes enunciados

(i) $E(t) \rightarrow 0$ cuando $t \rightarrow+\infty$.

(ii)El dominio $\Omega$ y los coeficientes $\lambda, \mu$ son tales que la única autofunción del sistema de Lamé (b) tal que (c) sucede, es la solución trivial $w \equiv 0$.

Por lo tanto, obtenemos el Teorema. 
Teorema 6.2 (Resultado Principal) Sea $\Omega$ un dominio suave, simplemente conexo, acotado de $\mathbb{R}^{3}$. Entonces, la solución de energía finita del sistema (1.1)-(1.3) es tal que $E(t) \rightarrow 0$ cuando $t \rightarrow+\infty$.

\section{REFERENCIAS}

[1] Andreou and Dassios -Dissipation of energy for magnetoelastic waves in a conductive medium Quart Appl. Math. LV(1) (1977), pp. 23-29.

[2] Avalos and Lassieka I.-exponential stability of a thermoelastic system with free boundary conditions without mechanical dissipation SIAM J. Math Anal. 29(1) (1998), pp. 155 -182 .

[3] Cortés L. -Resonant frequencies for a system of time-harmonic elastic wave J. Math. Anal. Appl. 279 (2003), pp. 43-55.

[4] Dafermos C. - On the existence and the asymptotic stability of solutions to the equations of lienar thermoelasticity. Arch. Rational Mech. Anal. 29 (1968), pp. 241 271.

[5] Haraux A. -Systemes dynamiques dissipatifs et applications Collet RMA. Vol 17, Masson, Paris, (1991).

[6] Kesavan S. -Topics in Functional Analysis and applications. John Wiley \& Sons, (1989).

[7] Koch H. -Slow decay in linear thermoelasticity (1997).

[8] Konopoff L. - The interaction betwenn elastic wave motions and a magnetic field in electrical conductors. J. Geophys Res 60 (1995), pp. 441-456.

[9] Kondratiev and Oleinik -Hardy's and Korn's type inequalities and theier applications. Rend. Mat. Appl. (7) 10 (1990), pp. 641 - 666.

[10] Pazy, A.-Semigroups of Linear Operators and Applications to Partial Differential Equations . Springer, New York, (1983).

[11] Y. Santiago A.-El Lema de Nakao y algunas aplicaciones. PESQUIMAT Revista de la Fac. CC. MM. de la UNMSM. Vol X Nro. 1, agosto (2007), pp. 33-44.

[12] Y. Santiago A. -Decaimiento exponencial de la solución débil de una ecuación de onda no lineal. PESQUIMAT Revista de la Fac. C. MM. de la UNMSM. Vol VIII Nro. 2, Diciembre (2005), pp. 29 - 43.

[13] Y. Santiago A. and J. Rivera - Global existence and exponential decay to the wave equation with localized frictional damping. PESQUIMAT Revista de la Fac. CC. MM. de la UNMSM. Vol V. Nro. 2, Diciembre (2002), pp. 1-19.

[14] Y. Santiago A. and J. M. Rivera -Exponential decay for a Von Kármán system with memory Advances in Differential Equations. Vol 9, numbers 9-10. September/October (2004). 
[15] Y. Santiago A. -Estabilidad exponencial del semigrupo $C_{o}$ asociado a un sistema Termoelástico. PESQUIMAT Revista de la Fac. CC. MM. de la UNMSM. Vol VII, Nro. 1, Junio (2004), pp. 30-42.

[16] Y. Santiago A. -Algunas desigualdades integrales y aplicaciones.(2006).

[17] Perla Menzala and E. Zuazua -Explicit exponential decay rates for solutuons of Von Kármáns system of thermoelastic plates. CR. Acad. Sci. Paris Ser I 3324 (1997), pp. $49-54$.

[18] Teman R. -Infinite-dimensional dynamical systems in Mechanics and Physic Springer. (1988). 